\title{
Use of Antibacterial Agents in an Intensive Care Unit in a Hospital in Brazil
}

\author{
E.F. dos Santos ${ }^{1}$, L. Lauria-Pires ${ }^{2}$, M.G. Pereira ${ }^{2,3}$, A.E. Silva ${ }^{2}$, I.P. Rodrigues ${ }^{4}$ and M.O. Maia ${ }^{5}$ \\ ${ }^{1}$ Postgraduate student of Medical Sciences in Brasília University; ${ }^{2}$ Medical School of Brasília University; ${ }^{3}$ Medical School, \\ Catholic University of Brasília; ${ }^{4}$ University Hospital of Brasília; ${ }^{5}$ Santa Luzia Hospital; Brasília, DF, Brazil
}

\begin{abstract}
It is essential to monitor the utilisation of antibacterial drugs in order to establish appropriate measures for their control. The pattern of usage of antibacterial drugs, and its association with indicators of hospital infection, has been investigated in a non-specialized adult intensive care unit (ICU) located in Santa Luzia Hospital (Brasília, DF, Brazil). The study was conducted between January 2001 and June 2004. Data concerning the utilisation of systemic antibacterial drugs, classified according to the Anatomical Therapeutic Chemical/Defined Daily Dose (ATC/DDD) system, and indicators of hospital infection, defined according to the National Nosocomial Infections Surveillance (NNIS) system, were obtained from appropriate hospital archives. During the study period, the average utilisation of antibacterial drugs was 1918.5 DDD units per 1000 patient-day $\left(\right.$ DDD $\left._{1000}\right)$. The three most used drugs were penicillins/beta-lactamase inhibitors $\left(535.3 \mathrm{DDD}_{1000}\right)$, third generation cephalosporins $\left(239.1 \mathrm{DDD}_{1000}\right)$ and quinolones $\left(212.5 \mathrm{DDD}_{1000}\right)$. The total utilisation of antibacterial drugs was correlated significantly with the incidence of hospital infection $(R=0.62 ; p<0.01)$ and the index of invasive procedures $(R=0.41 ; p<0.01)$. Furthermore, the latter two indicators were significantly and positively correlated with the use of recently commercialised, broad spectrum antibacterial drugs (except for carbapenems). It is concluded that improved infection control procedures, together with more rigorous criteria regarding the use of invasive procedures, should be implemented by the ICU studied in order to diminish the utilisation of antibacterial drugs.
\end{abstract}

Key Words: Antibacterial drugs, intensive care unit, hospital infections, invasive practices.

The discovery of antibiotic agents represents one of the most important milestones in the advancement of medical science since it has enabled infectious diseases including puerperal fever, pneumococcal pneumonia and bacterial meningitis, which had been responsible for enormous numbers of deaths, to be successfully treated and cured. On the other hand, the ubiquitous and excessive use, or even misuse, of antibiotics has contributed to the appearance of resistant strains of microorganisms, especially in those environments where such drugs are used extensively [1-3].

Various strategies have been proposed with the aim of moderating the emergence of bacterial resistance in the hospital environment [4-6]. However, the success of such measures depends largely on the availability of information regarding the form in which antibiotics are administered and salient factors relating to their excessive or inadequate use, particularly in ICUs. To the best of our knowledge, no published studies have evaluated the use of antibacterial drugs, as classified according to the Anatomical Therapeutic Chemical/Defined Daily Dose (ATC/DDD) model, in Brazilian ICUs [7]. Therefore, the objectives of the present study were to evaluate the pattern of usage of antibacterial drugs in an adult ICU in a general hospital in Brasilia - DF, Brazil, and to identify which indicators of hospital infections were related to the greater usage of these agents.

Received on 11 January 2007; revised 7 May 2007.

Address for correspondence: Dr. Edilson Floriano dos Santos. Fundação de Ensino e Pesquisa em Ciência da Saúde. SMHN Quadra 03, Conjunto A, Bloco 1, Edifício FEPECS. Zip code: 70.710-907, Brasília, DF, Brazil. Phone: +55 6133254956 Fax: +55 613326 5540. E-mail: edilson@ambr.com.br.

The Brazilian Journal of Infectious Diseases

2007;11(3):355-359. (C) 2007 by The Brazilian Journal of Infectious Diseases and Contexto Publishing. All rights reserved.

\section{Material and Methods}

The adult ICU investigated was a 20-bed medical-surgical unit of Hospital Santa Luzia, a private, non-specialised hospital with 140 beds providing general medical care for about 10,000 patients a year in Brasília - DF, Brazil. Data concerning all patients treated in the ICU during the period January 2001 to June 2004 were considered in the analysis: the aspects evaluated were (i) the utilisation of antibacterial agents (ii) the incidence of general and specific hospital infections, and (iii) the proportional use of invasive procedures.

\section{Determination of the Use of Antibacterial Drugs}

The classification of drugs followed the Anatomical Therapeutic Chemical/Defined Daily Dose" (ATC/DDD) system adopted by the World Health Organization (WHO).[7] In this system, drugs are categorised into various groups according to the organ or system upon which they act and according to their pharmacological and therapeutic properties. Defined daily dose (DDD) represents the average adult daily maintenance dose of a specific drug applied according to its primary indication. The quantity of each drug released by the hospital pharmacy to the ICU during a monthly period was transformed into units of DDD and divided by the number of patient-days in the month. This value was then multiplied by 1,000 so that the final figure $\left(\mathrm{DDD}_{1000}\right)$ represented the level of utilisation of an antibacterial drug per 1,000 patient-days.

Criteria Used for Assessing Indicators of Hospital Infections

In order to analyse hospital infections, the definitions established by $\mathrm{CDC}[8]$ were applied, in which such infections are considered as all of those conditions (localised or systemic) that result in a reaction of the organism to the presence of one or more infectious agents or toxins derived from them. Furthermore, the conditions developed by the patient must 
have been acquired after admission to the hospital and have emerged during the period of confinement or after the patient had been released. In addition, the type of infection must be related to the hospital environment or to the invasive procedures performed during confinement. Analyses were based on clinical and laboratory evidence, as well as on other tests including endoscopies, biopsies and needle aspirations. All patients admitted to the ICU were monitored for the presence of hospital infections in all areas of the body during their period of confinement. The performance of any invasive procedure (i.e. mechanical ventilation, use of central venous catheters and indwelling urinary catheters) was logged daily for each of the patients. Every month, the number of each specific invasive procedure conducted were totalled and the values divided by the total number of patient-days, thus providing an index of invasive procedures employed (device associated) in the ICU.

\section{Data Collection}

The data used in this study were obtained from two independent data bases maintained by the hospital, from which statistical summaries were produced at regular intervals. With respect to the distribution of antibacterial drugs, details of all medicines prescribed in the various sectors of the hospital were transcribed from the medical prescriptions written for individual patients and entered into the computer system by trained personnel. This archive thus contains information concerning the types and amounts of drugs administered to all patients during their period of stay within each sector of the hospital. In the present study, the target drugs were the systemic antibacterial agents belonging to class J01 of the ATC/DDD classification system. The systemic antimycotic (J02), the antimycobacterial (J04) and the systemic antiviral (J05) drugs were not included in the study.

Hospital Santa Luzia has installed an Infection Control Service, which comprises a team of specialised personnel (doctors, nurses, and medical assistants) whose function is to prevent and control infections. Information regarding the occurrence of infections and the execution of invasive procedures is collected daily by the team from the various sectors of the hospital. This information derives from physical visits by team members, from patients' records and from microbiological tests carried out on behalf of the team. All data collected are recorded in the data base on standard forms based on the NNIS model [9], and monthly summary reports are produced for each individual hospital sector.

\section{Statistical Analysis}

Continuous variables with parametric distributions (for example, level of utilisation of drugs) were compared by analysis of variance (ANOVA) following evaluation of their homogeneity determined using the Levene test. Continuous variables with non-parametric distributions were analysed using Kruskal-Wallis test. The degree of correlation was determined from the Pearson coefficient, and the level of statistical significance was established at $\mathrm{p}=0.05$. Data were stored and analysed using EPI INFO 6.04d (CDC, 2001) and SPSS for Windows 10.0.1 (SPSS Inc.) software.

\section{Ethical Considerations}

Details of the study were presented to and approved by the Ethical Committee of Hospital Santa Luzia.

\section{Results}

Evaluation of the Incidence of Hospital Infection

During the study period, about 2,890 patients were received in the adult ICU giving rise to a total of 15,410 patient-days. The number of patients who acquired hospital infections during this time was 283, resulting in an average of 18.4 cases of infection per 1,000 patient-days. Pneumonia, occasioned by the use of mechanical ventilation, was the most frequent type of infection encountered and was responsible for 97 cases per 5944 ventilator-days (16.3 cases per 1,000 ventilator-days). The second most common type of infection was of the bloodstream and was associated with the use of central vascular cannulation: this resulted in 51 cases per 8,927 central line-days (5.7 cases per 1,000 central line-days). Urinary infection, associated with the use of indwelling urinary catheters, resulted in 52 cases per 9,116 catheter-days (5.7 cases per 1,000 urinary catheter-days).

\section{Evaluation of the Usage of Antibacterial Drugs}

The average utilisation of antibacterial drugs in the studied ICU was 1,918.5 DDD per 1,000 patient-days (Table 1). The six most prescribed groups of antibacterial drugs were, in decreasing order: penicillin/beta-lactamase inhibitors, third generation cephalosporins, quinolones, carbapenems, glycopeptides and second generation cephalosporins. These drug groups, taken together, accounted for $72.3 \%$ of the average usage of antibacterials during the period of study. Considering each antibacterial agent individually in terms of their DDD ${ }_{1000}$ values, the most used were ampicillin/sulbactam (417.4), meropenem (140.4), vancomycin (131.3), ciprofloxacin (129.3) and ceftriaxone (128.3).

When the average utilisation of all antibacterial drugs, taken together, was evaluated for each year, it was observed that there were no significant alterations during the period 2001-2004 (Table 2). However, there was a significant increase in the average usage of penicillin/beta-lactamase inhibitors, mainly due to the increased prescription of ampicillin/ sulbactam and amoxicillin/clavulanic acid. On the other hand, there was a decrease in the average utilisation of glycopeptides. This reduction might have been associated with the introduction of linezolid, a drug that was only commercialised in Brazil in 2001, which exhibits activity against oxacillin-resistant Staphylococcus aureus. Thus, the evaluation took into account the average utilisation of the sum of glycopeptides and linezolid but, as demonstrated in Table 3, the average usage of these drugs taken together still showed a significant decrease during the period. 
Table 1. Utilisation of antibacterial drugs in the adult ICU of Hospital Santa Luzia, Brasília - DF, Brazil, during the period of January 2001 to June 2004

\begin{tabular}{lcc}
\hline \multirow{2}{*}{ Antibacterial group $^{\mathbf{a}}$} & \multicolumn{2}{c}{ Drug usage $^{\mathbf{b}}$} \\
\cline { 2 - 3 } & \multicolumn{1}{c}{ Mean (sd) } & ${\text { Median }\left(\mathbf{2 5}^{\text {th }} \mathbf{- \mathbf { 7 5 } ^ { \text { th } }} \text { percentile) }\right.}$ \\
\hline Beta-lactamase sensitive penicillins & $32.1(79.4)$ & $0.0(0.0-0.0)$ \\
Penicillins with extended spectrum & $5.6(21.3)$ & $0.0(0.0-0.0)$ \\
Beta-lactamase resistant penicillins & $84.1(132.3)$ & $36.4(1.1-106.6)$ \\
Penicillins/beta-lactamase inhibitors [1] & $535.3(299.4)$ & $525.3(356.0-708.6)$ \\
First generation cephalosporins & $80.7(50.0)$ & $67.6(43.2-100.1)$ \\
Second generation cephalosporins [6] & $120.6(121.0)$ & $96.6(48.2-166.4)$ \\
Third generation cephalosporins [2] & $239.1(133.1)$ & $205.1(147.5-299.0)$ \\
Carbapenems [4] & $155.1(70.2)$ & $161.0(114.4-207.0)$ \\
Aztreonam & $7.2(11.2)$ & $0.8(0.0-13.1)$ \\
Glycopeptides [5] & $145.2(84.0)$ & $136.2(88.1-182.7)$ \\
Quinolones [3] & $212.5(101.0)$ & $193.4(153.2-278.5)$ \\
Trimethoprim/sulphamethoxazole & $23.7(46.3)$ & $2.2(0.0-23.3)$ \\
Macrolides & $22.1(35.3)$ & $0.0(0.0-38.4)$ \\
Aminoglycosides & $95.4(52.3)$ & $83.4(51.9-127.1)$ \\
Imidazoles & $58.3(47.4)$ & $49.5(19.7-85.4)$ \\
Lincosamides & $88.7(63.4)$ & $73.2(0.0-147.6)$ \\
Linezolid & $41.4(48.7)$ & $22.2(0.0-78.5)$ \\
Total & $1,918.5(532.4)$ & $1,895.4(1,639.8-2,171.2)$ \\
\hline
\end{tabular}

${ }^{a}$ Classified according to the Anatomical Therapeutic Chemical/Defined Daily Dose (ATC/DDD) system: the numbers shown in square brackets indicate the ranking order of the six most prescribed antibacterial agents. ${ }^{b}$ Usage expressed in defined daily doses (DDD) per 1,000 patient-days calculated from (total number of DDD of a drug used / total number of patient-days) $\mathrm{x}$ 1,$000 ; \mathrm{sd}=$ standard deviation. ${ }^{\mathrm{c}}$ Total of antibacterial drugs in all groups.

Table 2. Evolution of the annual utilisation of the six most prescribed antibacterial drugs in the adult ICU of Hospital Santa Luzia, Brasília - DF, Brazil, during the period of January 2001 to June 2004

\begin{tabular}{|c|c|c|c|c|c|}
\hline \multirow[t]{2}{*}{ Antibacterial group ${ }^{a}$} & \multicolumn{4}{|c|}{ Drug usage $^{b}$} & \multirow[t]{2}{*}{ p-value } \\
\hline & 2001 & 2002 & 2003 & 2004 & \\
\hline Penicillins/beta-lactamase inhibitors & $306.6^{*}$ & 509.3 & $714.5^{*}$ & $686.5^{*}$ & 0.002 \\
\hline Third generation cephalosporins & 268.5 & 282.0 & 174.1 & 224.1 & 0.190 \\
\hline Quinolones & 158.0 & 238.4 & 245.5 & 203.8 & 0.130 \\
\hline Carbapenems & 140.2 & 148.3 & 177.8 & 153.3 & 0.605 \\
\hline Glycopeptides & 146.1 & $214.5^{*}$ & $112.3 *$ & $70.3^{*}$ & 0.001 \\
\hline Second generation cephalosporins ${ }^{c}$ & 66.1 & 200.5 & 102.3 & 106.5 & 0.086 \\
\hline Glycopeptides + linezolid & $149.3 *$ & $237.3 *$ & 190.9 & 150.8 & 0.024 \\
\hline Total $^{\mathrm{d}}$ & 1672.5 & 2156.1 & 1974.3 & 1824.1 & 0.151 \\
\hline
\end{tabular}

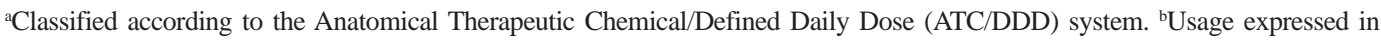
defined daily doses (DDD) per 1,000 patient-days calculated from (total number of DDD of a drug used / total number of patient-days) x 1,000. Mean values labelled * are statistically significantly different $(\mathrm{p} \leq 0.05)$ from each other on a year-byyear basis within the antibacterial group.'Kruskal-Wallis test (non-parametric). ${ }^{\mathrm{d}}$ Total of antibacterial drugs of all groups.

There were positive and significant correlations between the use of recently introduced broad spectrum antibacterial drugs and both the incidence of hospital infection and the index of invasive procedures (Table 3). The exception to this generalisation was for the carbapenems, the correlations of which were not significant.

\section{Discussion}

The results of the study revealed that each patient received, on average, almost two DDDs of antibacterial drugs per day during their period of confinement in the
ICU. Generally, the drugs employed were of broad spectrum activity, and factors related to the occurrence of nosocomial infections, such as incidence of hospital infection, ratio of the use of mechanical ventilation and long-term vesical catheters, were associated with the increased administration of these agents. The average global rate of hospital infection in the ICU was $9.8 \%$, indicating that the situation was under reasonable control. However, the rates of infection specifically associated with the use of invasive procedures (namely, pneumonia, bloodstream and urinary infections) were consistently 
Table 3. Correlation of the usage of antibacterial drugs with the incidence of hospital infection and with the index of utilisation of invasive procedures in the adult ICU of Hospital Santa Luzia, Brasília - DF, Brazil, during the period of January 2001 to June 2004

\begin{tabular}{|c|c|c|}
\hline \multirow[t]{2}{*}{ Antibacterial group $^{a}$} & \multicolumn{2}{|c|}{ Pearson correlation coefficient ${ }^{\text {b }}$} \\
\hline & Incidence of hospital infection ${ }^{c}$ & Index of utilisation of invasive procedures ${ }^{\mathrm{d}}$ \\
\hline $3^{\text {rd }}$ Generation cephalosporins & $0.42 * *$ & $0.37 *$ \\
\hline Quinolones & $0.42 * *$ & $0.32 *$ \\
\hline Carbapenems & 0.06 & 0.13 \\
\hline Glycopeptides & $0.52 * *$ & $0.51 * *$ \\
\hline Total $^{\mathrm{e}}$ & $0.62 * *$ & $0.41 * *$ \\
\hline
\end{tabular}

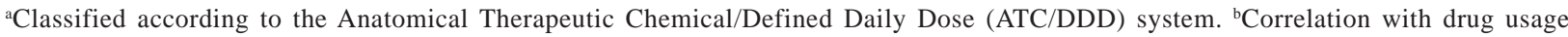
expressed in defined daily doses (DDD) per 1,000 patient-days calculated from (total number of DDD of a drug used / total number of patient-days) x 1,000. Correlation coefficients labelled *are statistically significant at $\mathrm{p} \leq 0.05$, whilst values labelled $* *$ are statistically significant at $\mathrm{p} \leq 0.01$. ${ }^{\mathrm{C}}$ Mean values of the variables related to the occurrence and risk of hospital infection. ${ }^{\mathrm{d}} \mathrm{Calculated}$ as the quotient of the total number of procedures carried out and the total number of patient-days. ${ }^{\text {eTtal }}$ of antibacterial drugs of all groups.

higher than those established in corresponding medicalsurgical ICUs that have adopted the NNIS system.[10]

\section{Pattern of Utilisation of Antibacterial Agents}

The median value of the overall usage of antibacterial drugs in the ICU of Hospital Santa Luzia (1895.4 DDD ${ }_{1000}$ ) was higher than those reported for interdisciplinary ICUs in hospitals in Germany (1252 DDD ${ }_{1000}$ )[11] and in Sweden (983 to $1391 \mathrm{DDD}_{1000}$ ) [12]. The six groups of antibacterial drugs most used in the Brazilian ICU were similar to those used in ICUs in Germany, with the exception that more glycopeptides were employed in Brazil. Compared with the practices followed in ICUs in Swedish hospitals, however, the differences regarding the use of antibacterial drugs were more accentuated. Thus, not only were glycopeptides used more frequently in Brazil, but the spectrum of activity of these drugs was different from those used in Sweden. Accordingly, whilst broad spectrum drugs such as ampicillin/sulbactam and meropenem were used most frequently in Hospital Santa Luzia, Swedish ICUs mainly utilised drugs with a narrower spectrum of activity such as cefuroxime and isoxazolylpenicillin [12].

The ICU of Hospital Santa Luzia employs a well designed strategy for controlling and rationalising antibacterial therapy, including revision and restriction of the use of antibacterial agents in the hospital, implementation of the guidelines for the empirical use of antimicrobials in the treatment of hospitalacquired pneumonias (the most frequent class of infection encountered in an ICU), and the requirement that a physician specialising in infectious diseases be consulted in relation to the antimicrobial therapy provided for patients with defined and/or suspected infection [13]. Despite such measures, however, the ICU was unable to achieve the standards followed in developed countries, and there are various possible explanations for this situation. In general terms, Brazil, as a developing country, can not provide adequate health care for the whole population and, therefore, access to intensive therapy is more restricted than in developed countries. Added to this, there are procedural and administrative difficulties regarding the acquisition of technological resources, including the recruitment of sufficient numbers of qualified personnel. Furthermore, there are cultural factors that need to be considered [14]. A more specific explanation would relate the pattern of usage of antibacterial drugs to numerous determinant factors including the frequency of occurrence of resistant microorganisms and the existence of, and adhesion to, methods for controlling and rationalising antibiotic therapy.

As a result of globalisation, the prevalence of microorganism resistance is similar in most countries since the therapeutic options are essentially the same. However, certain regions enjoy a low frequency of resistant microorganisms, as is the case for Swedish ICUs where methicillin-sensitive Staphylococcus aureus is prevalent [12]. Moreover, access to advice from consultants with particular expertise in specific infectious diseases may permit the optimisation of appropriate therapy and hence minimise the utilisation of drugs [13]. It is possible that the interaction between the frequency of bacterial resistance, the effective adhesion to control and rationalisation of therapy, and various other factors that are specific to the ICU (such as the type of patient admitted, availability of material and human resources, level of organisation) may account for the differences in the pattern of usage of antibacterial agents between the ICUs.

Factors Associated with the Usage of Antibacterial Agents

Antibacterial drugs should only be used when there is a confirmed infection or when the likelihood of the occurrence of an infection is high [15]. Since the majority of hospital infections are caused by bacteria, a positive correlation between the utilisation of antibacterial drugs and the risk or occurrence of infection is to be expected. In the analysis of such correlations, however, some further considerations must be taken into account. Firstly, drug resistance is higher amongst bacterial strains originating from hospitals in 
comparison with other environments, and hence the use of new generation antibacterial agents in their treatment is favoured. Secondly, patients in a critical condition require invasive procedures more often and, therefore, they are more vulnerable to hospital infection such that broad spectrum antibacterial agents are used from the beginning of the treatment. These arguments may explain the correlation of hospital infection rates and the index of invasive procedures with the usage of new generation broad spectrum antibacterial drugs. In the case of Hospital Santa Luzia, there was no correlation between the use of carbapenems and indicators of hospital infection, thus suggesting inappropriate use of these drugs.

\section{$\underline{\text { Advantages and Limitations }}$}

Evaluations of the nature presented in this study are useful for revealing the types of approach employed by particular ICUs with respect to their use of antibacterial drugs, for comparing the various schemes adopted by different institutions in different regions, for verifying the relationships between the utilisation of antibacterial drugs and the indicators of hospital infections, and for assessing the impact of the level of antibacterial drug usage on the occurrence of bacterial resistance. Despite their known value, however, these evaluations have some limitations. The data obtained do not permit the direct evaluation of the adequacy of antibiotic therapy for individual patients since inferences are based on relationships amongst variables with no allowance for any potential hidden factors. In the present study, the performance of just one ICU was evaluated and the results may reflect local practices as they take into account specific characteristics and ecology. The comparison of data between Brazil and developed countries, such as Germany and Sweden, is subject to systematic errors and is only useful for the establishment of initial parameters. The social and economic realities of developed countries are significantly different from those of developing countries such as Brazil, and thus the quality of prescriptions cannot be the judge on the strength of such comparisons.

\section{Conclusions}

In the present study, it has been demonstrated that an ICU in a Brazilian hospital used larger quantities of antibacterial drugs than ICUs in developed countries, and that such drugs were mostly of a broad spectrum of activity. The total utilisation of antibacterial drugs was associated with the global incidence of hospital infection in the unit as well as with the application of procedures that bypass the natural barriers of the patients against infection, thus increasing the risk of infection. The results suggest that the adoption of basic measures for the rationalisation of antibacterial therapy, such as the optimisation of the therapy, supervision by experts specialising in infectious diseases, and the follow-up of infected patients, are essential in order to diminish the utilisation of antibacterial drugs in the ICU. Measures designed to prevent the incidence of hospital infection (cleaning of hands and the adoption of barrier techniques) may need more rigorous enforcement. Finally, greater control over the application of invasive procedures would also contribute to the minimisation of the usage of antibacterial agents.

\section{References}

1. Archibald L., Phillips L., Monnet D., et al. Antimicrobial resistance in isolates from inpatients and outpatients in the United States: increasing importance of the intensive care unit. Clin Infect Dis 1997;24(2):211-5.

2. Fridkin S.K., Gaynes R.P. Antimicrobial resistance in intensive care units. Clin Chest Med 1999;20(2):303-16,viii.

3. Meyer E., Jonas D., Schwab F., et al. Design of a surveillance system of antibiotic use and bacterial resistance in German intensive care units (SARI). Infection 2003;31(4):208-15.

4. Goldmann D.A., Weinstein R.A., Wenzel R.P., et al. Strategies to prevent and control the emergence and spread of antimicrobialresistant microorganisms in hospitals. A challenge to hospital leadership. JAMA 1996;275(3):234-40.

5. Shlaes D.M., Gerding D.N., John J.F., Jr., et al. Society for Healthcare Epidemiology of America and Infectious Diseases Society of America Joint Committee on the Prevention of Antimicrobial Resistance: guidelines for the prevention of antimicrobial resistance in hospitals. Infect Control Hosp Epidemiol 1997; $18(4): 275-91$

6. World Health Organization's strategy to contain resistance to antimicrobial drugs. Rev Panam Salud Publica 2001;10(4):284-94.

7. Anatomical Therapeutic Chemical (ATC) classification index with Defined Daily Doses (DDDs). Oslo (Norway): WHO Collaborating Centre for Drug Statistics Methodology. 2005.

8. Garner J.S., Jarvis W.R., Emori T.G., et al. CDC definitions for nosocomial infections, 1988. Am J Infect Control 1988; $16(3): 128-40$.

9. Emori T.G., Culver D.H., Horan T.C. National nosocomial infections surveillance system (NNIS): description of surveillance methods. Am J Infect Control 1991;19(1):19-35.

10. National Nosocomial Infections Surveillance (NNIS) System Report, data summary from January 1992 through June 2004, issued October 2004. Am J Infect Control 2004;32(8):470-85.

11. Meyer E., Schwab F., Jonas D., et al. Surveillance of antimicrobial use and antimicrobial resistance in intensive care units (SARI): 1. Antimicrobial use in German intensive care units. Intensive Care Med 2004;30(6):1089-96.

12. Hanberger H., Erlandsson M., Burman L.G., et al. High antibiotic susceptibility among bacterial pathogens in Swedish ICUs. Report from a nation-wide surveillance program using TA90 as a novel index of susceptibility. Scand J Infect Dis 2004;36(1):24-30.

13. Dos Santos E.F., Silva A.E., Pinhati H.M., Maia M.O. Effectiveness of the actions of antimicrobial control in the intensive care unit. Braz J Infect Dis 2003;7(5):290-6.

14. Soriano F.G. APACHE and Brazilian intensive care units. Sao Paulo Med J 2003;121(2):43-4.

15. Moellering Jr. R.C., Eliopoulos G.M. Principles of anti-infective therapy. In: Mandell GL, Bennett JE, Dolin R, eds. Principles and Practice of Infectious Diseases. 6th ed. Philadelphia, Pennsylvania: Elsevier, 2005. 